\title{
Ground-Based GPS Tomography of Water Vapor: Analysis of Simulated and Real Data
}

\author{
Lubomir P. GRADINARSKY \\ Onsala Space Observatory, Onsala, Sweden \\ and \\ Per JARLEMARK \\ SP Swedish National Testing and Research Institute, Sweden \\ (Manuscript received 16 March 2003, in revised form 7 November 2003)
}

\begin{abstract}
We present the concept, some of the approaches used, and the capabilities of the technique referred to as GPS tomography. It is used for retrieval of the 3-dimensional distribution of the refractivity due to atmospheric water vapor. We discuss the presently used methods for retrieval of the primary observable in the GPS tomography, the slant path delay, as well as their shortcomings. Comparisons of GPS slant delays to independent data from a microwave radiometer are included. From a tomographic point of view we concentrate on the capabilities to retrieve the vertical structure of the wet refractivity. For this purpose we present and apply two methods for tomographic inversion. Both are based on the Kalman filtering technique, where the expected statistical behavior of the refractivity is utilized. The difference between the two is in the way the covariance matrix of the Kalman filter is constructed. We base our study on simulated and real data from the ground network of 8 GPS receivers operating in Göteborg, Sweden. The results demonstrate that at present the limitations of the GPS tomographic technique are errors in the retrieved wet slant delays and their poor geometric distribution.
\end{abstract}

\section{The tomographic technique}

A subset of the inverse theory utilizing measurements of integrals of a certain field obtained from different angles and positions is referred to as tomography. Inversion of the measured integral values results in estimates of the field values in selected representative points. The main tomographic applications are in medicine (Jain 1989) and in seismology (Nolet 1987). In the medical applications of tomography 2 - and 3-D images are successfully reconstructed by utilizing a source-detector assembly rotating around an object to obtain

Corresponding author: Lubomir P. Gradinarsky, Onsala Space Observatory, 43992 Onsala, Sweden

E-mail: Ibg@oso.chalmers.se

(C) 2004, Meteorological Society of Japan projection views from different angles. This well controlled measurement environment provides opportunity for high-resolution image reconstruction. In seismologicaltomography applications, there is generally less control on the source-detector geometry. It has however the advantage that the parameter field and the detector locations do not change dramatically with time. This time stability provides opportunity for certain level of grid optimization according to the particular measurement set up. By modifying the grid accordingly we can increase the tomographic resolution (using smaller grid point separations) at locations where more observations cross and reduce the resolution (using larger grid point separations) for areas covered less by observations (Böhm and Vesnaver 1999). 


\section{GPS tomography}

By utilizing a number of GPS receivers forming a local network and assuming that observations of the delay along the propagation paths towards the different satellites are available (from now on referred to as slant delays) one could attempt to estimate not only the integrated values of the parameters affecting the delay, but can also obtain some information on their 3-D distribution. In this paper, we will discuss retrieval of the average refractivity in $3-D$ boxes referred to as voxels (volume pixels). First results of this approach, referred to as GPS tomography, have been obtained and retrievals of the vertical distribution of refractivity due to water vapor (Flores et al. 2001) and the Total Electron Content (Ruffini et al. 1998) were demonstrated.

\section{The observations}

The widely used approach to form the GPS tomographic observations, the wet slant delays (SD), utilizes the estimated tropospheric parameters from the post processed GPS data i.e. the zenith wet delay $\Delta L_{w}^{z}$, and the delay gradient terms $\Xi_{n}$ and $\Xi_{e}$, as well as the processing residual $\mathfrak{R}$. We can write the wet slant delay as a function of azimuth $\phi$ and elevation $\varepsilon$ :

$$
\begin{aligned}
\Delta L_{w}(\phi, \varepsilon)= & \Delta L_{w}^{z} \cdot m_{w}(\varepsilon)+\frac{d m_{w}(\varepsilon)}{d \varepsilon}\left[\Xi_{n} \cos (\phi)\right. \\
& \left.+\Xi_{e} \sin (\phi)\right]+\mathfrak{R}(\varepsilon, \phi)
\end{aligned}
$$

where $m_{w}(\varepsilon)$ is the wet mapping function (Niell 1996) and the gradient parameters $\Xi_{n}$ and $\Xi_{e}$ are defined by Davis et al. (1993). The processing residual term $\mathfrak{R}(\varepsilon, \phi)$ contains mainly the model error of the receiver clock, multipath, antenna phase center variations, and errors due to not modeled atmospheric variability. If we could minimize the impact of the first three of the above effects, $\mathfrak{R}(\varepsilon, \phi)$ will mainly contain only the atmospheric variability. Alber et al. (2000) suggested a method of obtaining the slant delays using the Double Difference processing method, which eliminates common satellite and receiver clock errors. The method is based on the assumption of zero mean single and double difference residuals. Braun et al. (2001) demonstrated an approach to reduce the effect of the antenna phase center variations through residuals stacking. This procedure can also reduce the systematic effect arising from multipath. Atmospheric parameters and residuals produced by using the Precise Point Positioning (PPP) technique (Zumberge et al. 1997) can also be used for retrieval of the slant delays. The shortcoming of all these SD reconstructions is that together with the atmospheric parameters, a large number of other parameters are also estimated simultaneously in the GPS analyses. Any atmospheric irregularity beyond the 3 parameter atmospheric modeling will be partly absorbed by the other estimated parameters, e.g. station clock, therefore limiting the SD estimation capabilities. This fundamental constraint was demonstrated by Elòsegui and Davis (2003) for the case of the Double Difference approach.

The potential information in the residual terms has been studied by Braun et al. (2001). They concluded that $\mathfrak{R}$ can contain the true atmospheric variability if this variability exceed the GPS noise levels. Their estimates of the GPS noise levels (expressed as delay) and using the Double Difference approach were between $6.5 \mathrm{~mm}$ RMS at $10^{\circ}$ to $15^{\circ}$ and below $1 \mathrm{~mm}$ at elevation angles of $85^{\circ}$ to $90^{\circ}$. They also demonstrated that the RMS of the estimated $\mathfrak{R}$ using Double Difference solution were approximately twice smaller for all elevations in comparison to the PPP solution. This was explained with the elimination of the receiver clock errors in the Double Difference approach.

Emardson et al. (2000a) described a 30-day experiment, where a pointed Water Vapor Radiometer (WVR) was used for comparison purposes, in an attempt to estimate the quality of the GPS derived wet slant delays. The PPP processing strategy was used. Comparison of the GPS derived tropospheric irregularity contribution (the $\mathfrak{R}$ term) with the WVR derived values demonstrated an RMS agreement of $11 \mathrm{~mm}$ with a correlation coefficient of 0.51 for the whole campaign with somewhat better correlation in certain periods of the experiment.

Below we present additional results on the agreement of the GPS obtained wet slant delay with data from a WVR. For this purpose we have performed a 30-day WVR experiment at Onsala, where we used 17 days with no data gaps or processing problems. The WVR schedule was designed so that it pointed towards each of the GPS satellites in view every 5 min- 

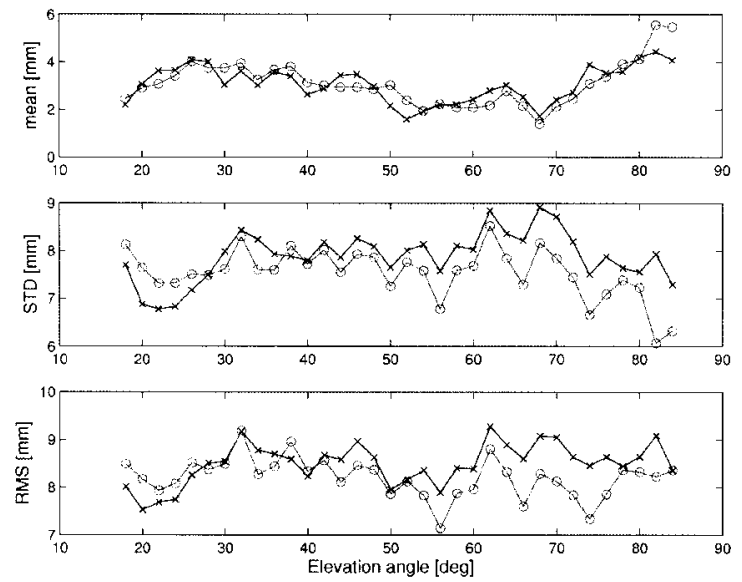

Fig. 1. The mean (GPS-WVR), the STD, and the RMS difference of slant delays measured by GPS and WVR. The slant delay differences are mapped to zenith and are presented as a function of elevation. The results are based on the fiducial ( $\mathrm{x}$ ) and the non-fiducial (o) GPS solutions.

utes, in this way matching the GPS sampling time. We used the PPP approach and tested two different processing strategies using a fiducial (fixed coordinates) and a non fiducial (not fixed coordinates) approach. Fig. 1 presents the mean, the standard deviation (STD), and the root mean square (RMS) difference between the estimated slant delays from GPS and WVR as a function of the elevation angle for both types of solutions. The difference is mapped to zenith. We observe that the mean difference is around $3 \mathrm{~mm}$, consistent with the known bias between the GPS and WVR techniques (Emardson et al. 2000b and Gradinarsky et al. 2002), decreasing with elevation until up to $\approx 70^{\circ}$ but increasing above. The STD is decreasing at elevation angels above $\approx 70^{\circ}$, which produces the RMS difference to be more or less elevation independent. We should consider that these differences are mapped to zenith and therefore observations at low elevations will differ more than in the zenith direction. We used the above result to model the expected slant delay errors in our simulation analysis, where we use an elevation dependent (cosecant) error with an RMS of $8 \mathrm{~mm}$ in the zenith direction.

From Braun et al. (2001), Emardson et al. (2002a) and our experiment described in more details in Gradinarsky (2002), the general conclusion is that the wet zenith delay and the two linear gradient terms (Eq. 1) carry the main information about the state of the atmosphere. The residuals can only contribute when the true wet refractivity signal along the propagation path exceeds the technique's noise level, where the retrieved SD will still be in error due to the fundamental limits described above and in Elòsegui and Davis (2003).

Nevertheless, in this paper we will evaluate the tomographic method using SD derived using Eq. 1 and the PPP processing technique. The assumption of the three parameter description used in the model in Eq. 1 could hold even if an inversion event is present in the refractivity profile, ensuring that correct values for the SD could be obtained. However we still emphasize that alternative ways for retrieval of SD should be developed.

\section{GPS tomographic methods}

The main difficulties that the ground-based GPS tomography is facing today are the limited geometry of the scanning rays and the noise in the slant delay observations. We will generally have a poor and changing geometry dependent on the GPS satellite constellation, a dynamically changing refractivity field, and noisy measurements. Due to these reasons, special methods adapted to the case of GPS tomography have to be developed. In our study a Kalman Filtering (KF) technique (Brown and Hwang 1997) was adopted due to its good capability to estimate dynamically changing parameters. In this section we will present two of the approaches, which are used for the tomographic inversion of simulated and real GPS data. The Wet Refractivity Kalman Filter (WeRKaF) was developed in our group while the LOcal Tropospheric TOmography Software (LOTTOS) was originally developed and implemented by Flores et al. (2001). The main difference of the two approaches is in the design of the covariance matrix of the parameters' driving noise used in their KF implementations. We will discuss the derivation and the verification of the WeRKaF method in more details, but be less specific in the LOTTOS case due to the existing reference material e.g. Flores et al. (2001) and Ruffini et al. (1998). 


\subsection{The wet refractivity Kalman filter}

We have based our analysis on the expected statistical properties of the wet refractivity variations as derived from radiosonde (RS) data. Knowledge of these statistical properties of the wet refractivity will be exploited in the design of the covariance matrix used by the Kalman filter.

Following Treuhaft and Lanyi (1987) we can define a spatial Structure Function $D_{\chi}(\vec{r}, \vec{R})$ of the refractivity $\chi(\chi=$ index of refraction -1$)$, as:

$$
D_{\chi}(\vec{r}, \vec{R})=E\left\{[\chi(\vec{r}+\vec{R})-\chi(\vec{r})]^{2}\right\}
$$

where $\vec{r}$ and $\vec{r}+\vec{R}$ denote the locations of the 2 points having the same geopotential height and refractivities $\chi(\vec{r})$ and $\chi(\vec{r}+\vec{R})$, respectively. Assuming that $D_{\chi}(\vec{r}, \vec{R})$ depends only on $R=|\vec{R}|$ and using the modified result on the wet refractivity SF given in Treuhaft and Lanyi (1987) we can write:

$$
D_{\chi}(R)=\frac{R^{2 / 3}}{1+(R / L)^{2 / 3}} C^{2}
$$

where $C=2.4 \cdot 10^{-7} \mathrm{~m}^{-1 / 3}$ and $L=3 \cdot 10^{6} \mathrm{~m}$. This model is developed for points within the first kilometer from the ground. In our tomographic refractivity modeling we will need $D_{\chi}(R)$ to include a height dependence term, which we will achieve by modifying the constant $C$.

In the Kalman filter approach, the model for the refractivity in each voxel assumes a random walk behavior. Therefore we can write the refractivities in voxels $i$ and $j$ and for consecutive samples $k$ and $k+1$ as:

$$
\begin{aligned}
& \chi_{i}(k+1)=\chi_{i}(k)+w_{i}(k) \\
& \chi_{j}(k+1)=\chi_{j}(k)+w_{j}(k)
\end{aligned}
$$

where $w$ is the model driving noise with statistical properties in which for our case will be derived below. The true horizontal distance between the voxels is $R$. We introduce a new distance relating the voxels separated in time through equivalent distance representation. Assuming a sampling interval of $T$ and a wind speed of $V$ with a direction perpendicular to the vector of the ground distance between the voxels, we define the following spacing between voxels $\chi_{i}$ and $\chi_{j}$ at times $k$ and $k+1$ (see Fig. 2 for illustration):

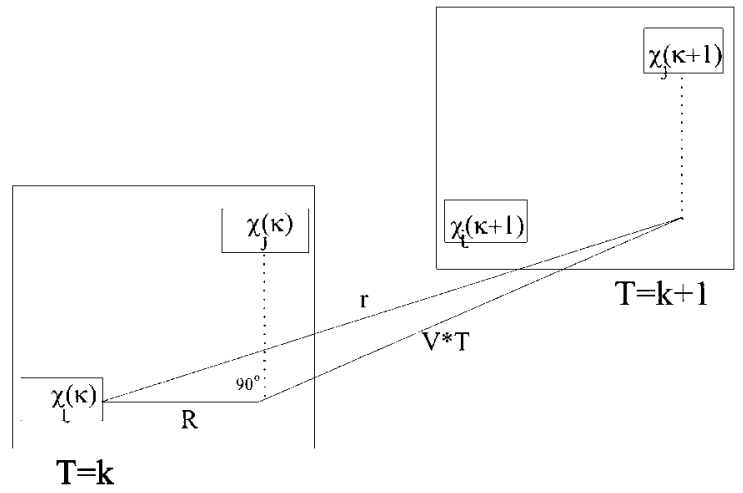

Fig. 2. Illustration of the assumption used for the derivation and the verification of the model in Eq. 9. The big boxes represent the voxel space at two consecutive time samples, separated by time $T$. The small boxes correspond to the $i$-th and $j$-th voxels having heights $h_{1}$ and $h_{2}$ separated geometrically by ground distance $R$ with their refractivities, respectively.

- True geometrical distance between voxels $\chi_{i}(k)$ and $\chi_{j}(k)$ which is given by $R_{3 D}^{2}=$ $R^{2}+\left(h_{i}-h_{j}\right)^{2}$ is modified to:

$$
\tilde{R}_{3 D}^{2}=R^{2}+C_{o} \cdot\left(h_{i}-h_{j}\right)^{2}
$$

where the constant $C_{o}$ will give additional weight for the separations in height in order to compensate for the expected greater variability in this dimension.

- Modified distance between voxels $\chi_{i}(k)$ and $\chi_{j}(k+1)$ is

$$
\tilde{R}_{4 D}^{2}=\tilde{R}_{3 D}^{2}+(V \cdot T)^{2}
$$

Using the SF definitions and $\tilde{R}_{3 D}$ as defined above, the result for the SF for points at different heights can be expressed as (Gradinarsky 2002):

$$
\begin{aligned}
D_{\chi}\left(h_{i}, h_{j}, R\right)= & m^{2}\left(h_{i}\right)+m^{2}\left(h_{j}\right) \\
& +\frac{\tilde{R}_{3 D}^{2 / 3}}{1+\left(\tilde{R}_{3 D} / L\right)^{2 / 3}} C^{2} e^{-\left(h_{i}+h_{j}\right) / h_{s c}}
\end{aligned}
$$

where $m^{2}\left(h_{i}\right)$ and $m^{2}\left(h_{j}\right)$ are the mean refractivities at heights $h_{i}$ and $h_{j}$, and $h_{s c}$ is a scale height.

Using the above definitions, the covariance between the variability of the refractivity in 
voxels $\chi_{i}$ and $\chi_{j}$ needed for the Kalman Filter implementation can be shown to be given as (Gradinarsky 2002):

$$
\begin{aligned}
Q_{i j}= & E\left[w_{i} w_{j}\right] \\
= & E\left[\left(\chi_{i}(k+1)-\chi_{i}(k)\right)\left(\chi_{j}(k+1)-\chi_{j}(k)\right)\right] \\
= & 0.5 \cdot\left[D_{\chi}\left(\tilde{\boldsymbol{R}}_{4 D}\right)-D_{\chi}\left(\tilde{\boldsymbol{R}}_{3 D}\right)+D_{\chi}\left(\tilde{R}_{4 D}\right)\right. \\
& \left.\quad-D_{\chi}\left(\tilde{\boldsymbol{R}}_{3 D}\right)\right] \\
= & D_{\chi}\left(\tilde{\boldsymbol{R}}_{4 D}\right)-D_{\chi}\left(\tilde{\boldsymbol{R}}_{3 D}\right)
\end{aligned}
$$

where we used the equality $(a-b)(c-d)=$ $0.5 *\left[(a-d)^{2}-(a-c)^{2}+(b-c)^{2}-(b-d)^{2}\right]$. This is the most important result for our $\mathrm{KF}$ implementation, which will substitute all of the constraint equations used in the LOTTOS approach as we will discuss later. In this way we avoid the difficult process of tuning the constraints' weights used there. The final form of the noise covariance matrix $Q_{i j}$ used in the WeRKaF implementation is:

$$
\begin{aligned}
Q_{i j}= & D_{\chi}\left(h_{i}, h_{j}, \tilde{R}_{4 D}\right)-D_{\chi}\left(h_{i}, h_{j}, \tilde{R}_{3 D}\right) \\
= & {\left[\frac{\tilde{R}_{4 D}^{2 / 3}}{1+\left(\tilde{R}_{4 D} / L\right)^{2 / 3}}-\frac{\tilde{R}_{3 D}^{2 / 3}}{1+\left(\tilde{R}_{3 D} / L\right)^{2 / 3}}\right] } \\
& \cdot C^{2} e^{-\left(h_{i}+h_{j}\right) / h_{s c}}
\end{aligned}
$$

The model above has been verified using RS data from the Landvetter airport located within $25 \mathrm{~km}$ from Göteborg and thus within the tomographic 3-D space in our analyses. The RS were launched every 6 hours and we used 4 years of data. We studied the cases when two consecutive RS launches represented one column of the tomographic voxel space separated in time by 6 hours, thus recreating a 6 hour sampling. Using this assumptions we recreate the case when $R=0$, which in Fig. 2 corresponds to the situation when the $i$ and $j$ voxels are above each other producing: $\tilde{R}_{3 D}^{2}=C_{o}$. $\left(h_{i}-h_{j}\right)^{2}$ and $\tilde{R}_{4 D}^{2}=C_{o} \cdot\left(h_{i}-h_{j}\right)^{2}+(V \cdot T)^{2}$. Even though the time scale of 6 hour is much longer than the sampling rate of 5 min used in the tomographic analyses, this was one way to verify the model given by Eq. 9 and calculate the model constants especially after the fact that other suitable data are difficult to find. By calculating $Q_{i j}$ from the RS and fitting the result to Eq. 9, assuming a wind speed of $8 \mathrm{~m} / \mathrm{s}$ (an average value for the the studied area), we found the optimum values of the constants for this time scales to be $C=2.2 \cdot 10^{-7}$ and $C_{0}=$ 100 (see also Gradinarsky 2002 for further details and comparisons).

Even though the wind speed and direction differ greatly in height and in time, the assumption of $8 \mathrm{~m} / \mathrm{s}$ is used as a representative value for the region and for all heights. It is based on the studied RS profiles. We believe that this and the assumption for perpendicularity are appropriate for estimation of an average of the constants' values in Eq. 9. Since we analyzed a large $\mathrm{RS}$ data set an average representative wind speed is appropriate. As we will comment below exact knowledge of the $C$ and $C_{0}$ values for particular atmospheric conditions will not play the most crucial role in the $\mathrm{KF}$ performance, but certainly will help. We think that further work in this direction will be beneficial. The model for $Q_{i j}$ has not been verified for shorter spatio-temporal scales due to lack of suitable data for this purpose. However, based on our simulation studies of the KF performance, we found that the observation noise and the sites-satellites geometry are the most important factors for successful data inversion, while the exact knowledge of the $\mathrm{KF}$ constraints, at this stage, play a less significant role in the retrieval precess. Therefore in our $\mathrm{KF}$ setup we used the above model.

\subsection{The LOcal Tropospheric TOmography Software}

LOTTOS has a different KF implementation from WeRKaF. The difference is in the design of the observation equations, which impacts the structure of the measurements' covariance matrix $\mathbf{R}$ and also in the design of the driving noise covariance matrix $\mathbf{Q}$. The implementation of the $\mathrm{KF}$ observation equations $\left(\mathbf{z}_{\mathbf{k}}=\mathbf{H}_{\mathbf{k}} \mathbf{x}_{\mathbf{k}}+\mathbf{v}_{\mathbf{k}}\right)$ in LOTTOS is given by:

$$
\left|\begin{array}{c}
\mathbf{z}_{\mathbf{k}} \\
\mathbf{l}_{\mathbf{k}}
\end{array}\right|=\left|\begin{array}{l}
\mathbf{H}_{\mathbf{k}} \\
\mathbf{B}_{\mathbf{k}}
\end{array}\right| \mathbf{x}_{\mathbf{k}}+\left|\begin{array}{c}
\mathbf{v}_{\mathbf{k}} \\
\mathbf{0}
\end{array}\right|
$$

where $\mathbf{H}_{\mathbf{k}}$ is the mapping between the unknown parameters $\mathbf{x}_{\mathbf{k}}$ (refractivity in our case) and the observations (slant delays); $\mathbf{v}_{\mathbf{k}}$ is the observation noise; the additional terms $\mathbf{B}_{\mathbf{k}}$ and $\mathbf{l}_{\mathbf{k}}$ are referred to as constraint equations introducing certain degree of correlation between the parameters $\mathbf{x}_{\mathbf{k}}$. In LOTTOS there are 3 different sets of constraints: 
- The horizontal constraint-assumes a certain degree of similarity between the horizontally neighboring voxels. It is implemented as a Gaussian weighted mean with a controllable width.

- The vertical constraint-assumes a certain degree of similarity between the vertically neighboring voxels. It is implemented in two versions: (1) as a Gaussian weighted mean with a controllable width and (2) as exponentially increasing correlation coefficient between the voxels in one vertical column.

- The boundary constraint-assumes zero refractivity above a certain height.

The constraints can be regarded as additional observations having a covariance matrix $\mathbf{I} / \lambda$, where the values of $\lambda$ could be different for the different sets of constraints. For details of the LOTTOS method see Flores et al. (2001) and Ruffini et al. (1998).

\section{Tomographic results}

We present results based on the permanent GPS network operating in Göteborg, Sweden. We study the estimation capabilities of both the LOTTOS and the WeRKaF approaches. For this purpose we use simulated profiles of refractivity as well as inversions of real GPS slant delays and compared to refractivity profiles from radiosonde data.

\subsection{The Göteborg network}

The existing permanent network in Göteborg consists of eight GPS sites, four of which are used for differential GPS positioning while the other four were established for tomographic studies. Table 1 summarizes the site information and Fig. 3 displays their locations. The network baseline lengths are in the range 5 to $15 \mathrm{~km}$. The GPS antennas are mounted on the roofs of buildings, in a manner assuring good signal reception also at low elevation angles. The data download is automatic at each site.

\subsection{Data analysis}

To assess the capabilities of the Göteborg network, we performed simulations as well as analysis of real data. The general conditions applying for all of the presented cases were: 5 minutes sampling rate for the WeRKaF solutions and 15 minutes for LOTTOS. Normally a one day solution was run, where the KF was
Table 1. Permanent GPS sites in Göteborg. Our group established the top four while the rest belong to the Göteborg municipality.

\begin{tabular}{|c|c|c|c|c|}
\hline $\begin{array}{l}\text { GPS site } \\
\text { name }\end{array}$ & Lat. $\left[{ }^{\circ}\right]$ & Lon. $\left[^{\circ}\right]$ & $\begin{array}{l}\text { Height } \\
{[\mathrm{m}]}\end{array}$ & $\begin{array}{l}\text { Rec. } \\
\text { Type }\end{array}$ \\
\hline Chalmers & 57.688 & 11.979 & 114.13 & TR 8000 \\
\hline $\begin{array}{l}\text { Chalmers- } \\
\text { Lind. }\end{array}$ & 57.706 & 11.937 & 58.71 & Ash. Z12 \\
\hline Sisjön & 57.640 & 11.953 & 54.08 & Ash. Z18 \\
\hline $\begin{array}{l}\text { Saab- } \\
\quad \text { Ericsson }\end{array}$ & 57.677 & 12.019 & 151.75 & TR 8000 \\
\hline Göteborg & 57.707 & 11.965 & 56.80 & Leica \\
\hline Kortedala & 57.762 & 12.037 & 170.07 & Leica \\
\hline Biskopsg & 57.725 & 11.892 & 131.37 & Leica \\
\hline Frölunda & 57.650 & 11.912 & 112.64 & Leica \\
\hline
\end{tabular}

$* \mathrm{TR}=$ Turbo-Rogue; Ash. $=$ Ashtech.

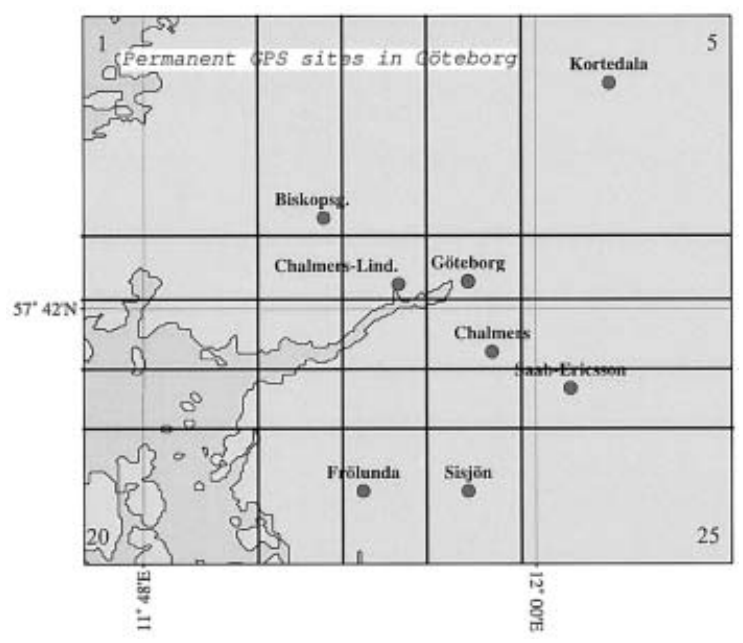

Fig. 3. The location of the permanent GPS sites in the Göteborg area. Displayed are also the voxel limits and the numbering used in the $5 \times 5$ voxel space. The voxel numbers increase from left to right and from top to bottom. The shortest baseline is between the sites Chalmers-Lind. and Göteborg, being $5 \mathrm{~km}$.

run in forward and backward mode. The elevation cut-off in both the simulated and the real data was 15 degrees. The simulation used one day data based on a real GPS satellite constellation. To all of the simulated data a NorthEast horizontal gradient (Davis et al. 1993) of $1 \mathrm{~mm}$ was superimposed to the data. The ex- 

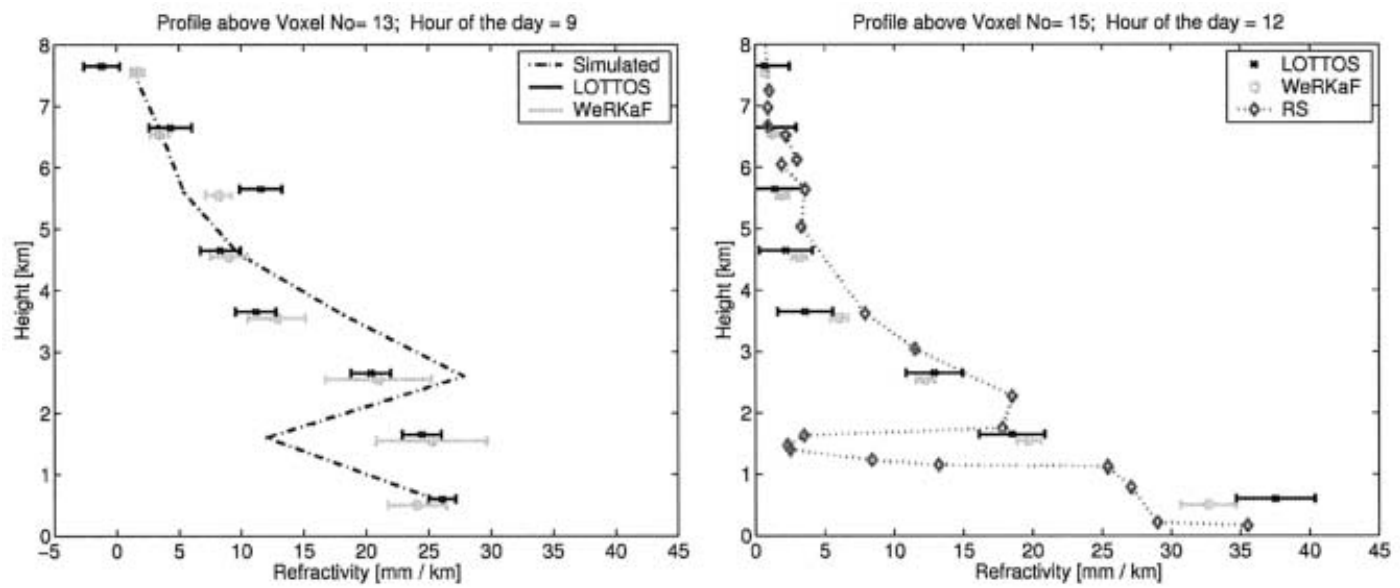

Fig. 4. Examples of estimated simulated (left) and real (right) refractivity fields from the Göteborg network using $5 \times 5 \times 8$ voxel space. Displayed are simulated/real profile and the corresponding LOTTOS and WeRKaF solutions. The presented solutions above voxel 13, being in the center of the grid space, and above voxel 15, where the launches of the used radiosonde occurred. The observation noise used in the simulation is elevation dependent with an RMS $=8 \mathrm{~mm}$. The corresponding RMS errors for the simulation are $4.7 \mathrm{~mm} / \mathrm{km}$ for LOTTOS and $4.2 \mathrm{~mm} / \mathrm{km}$ for WeRKaF. For the estimated real refractivity profile the corresponding RMS error when comparing to the the displayed RS profile is $7 \mathrm{~mm} / \mathrm{km}$ for both methods.

amples of the real data results are from May 2002.

Fig. 4 (left) is an example of simulated refractivity based on the real RS profile from Fig. 4 (right). The estimated values are based on the two approaches LOTTOS and WeRKaF. We used the observation error model obtained in the previous section. Fig. 4 (right) is the refractivity profile estimated from real GPS data from the $13^{\text {th }}$ of May 2002. The displayed solution is the one coinciding with the RS launch at 12 UT. The voxel space used was $5 \times 5 \times 8$ producing $1 \mathrm{~km}$ vertical resolution and uneven horizontal resolution from a few $\mathrm{km}$ in the center to some tens of kilometers in the boundary volume (see Fig. 3). We display the solutions above voxel 13 , being in the center of the grid space, and above voxel 15 , where the launches of the radiosondes used occurs. The estimation error for both cases is of the order of 4-7 mm/ $\mathrm{km}$. In the simulated and in the real data case, both tomographic retrieval methods give an average of the profile but fail to reconstruct the details of the vertical structure. The reason for that is a combination of a poor network geometry and the noise presence in the SD data. We note that the case studied is a difficult profile to estimate due to its extreme height variation. The boundary voxels also produced slightly larger estimation errors due to the poorer ray coverage.

In the cases of more regular profiles and inversion events occurring at lower heights as well as in presence of less noise, the tomographic results are closer to the simulated refractivities e.g. in Fig. 5 and in Gradinarsky and Jarlemark (2002). In Fig. 5, we simulated 9 hours of data (6 to 15 UT) using a real satellite constellation above the network. We simulated a situation where an average for the area refractivity profile was used for the time 6 to 9 UT. At 9 UT an increase began to occur at $1.5 \mathrm{~km}$ height. It reached its maximum at $12 \mathrm{UT}$ and then decreased. Fig. 5 (left) is the 9 UT profile and the estimates, while Fig. 5 (right) presents the 12 UT result. To the SD observations we added elevation independent Gaussian noise with an RMS of $10 \mathrm{~mm}$. We can see the WeRKaF inversion method in this situation was able to follow the simulated profile. We can also point out that WeRKaF generally performed better than LOTTOS and was also less sensitive to observation noise (Gradinarsky 2002). 

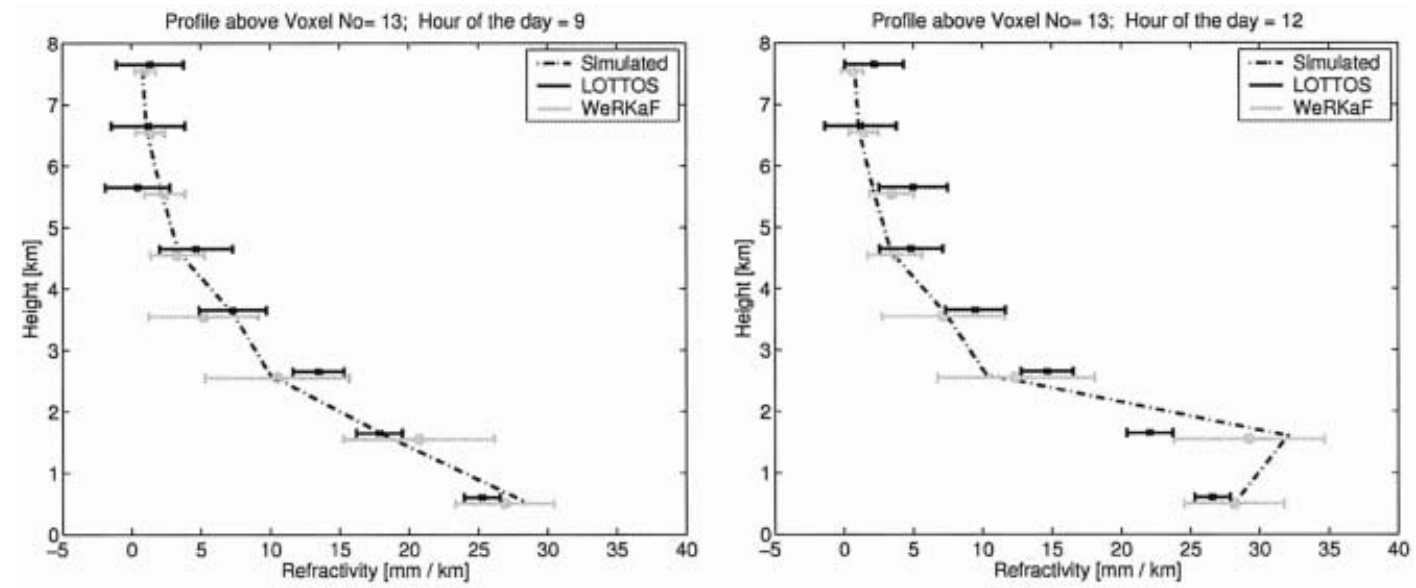

Fig. 5. Examples of estimated simulated refractivity fields using $5 \times 5 \times 8$ voxel space. The presented solutions are above voxel 13, being in the center of the grid space. Displayed are the simulated truth, the LOTTOS estimates and the WeRKaF estimates for: (left) a regular profile and (right) profile containing inversion. In both cases elevation independent Gaussian noise with an RMS of $10 \mathrm{~mm}$ was added.
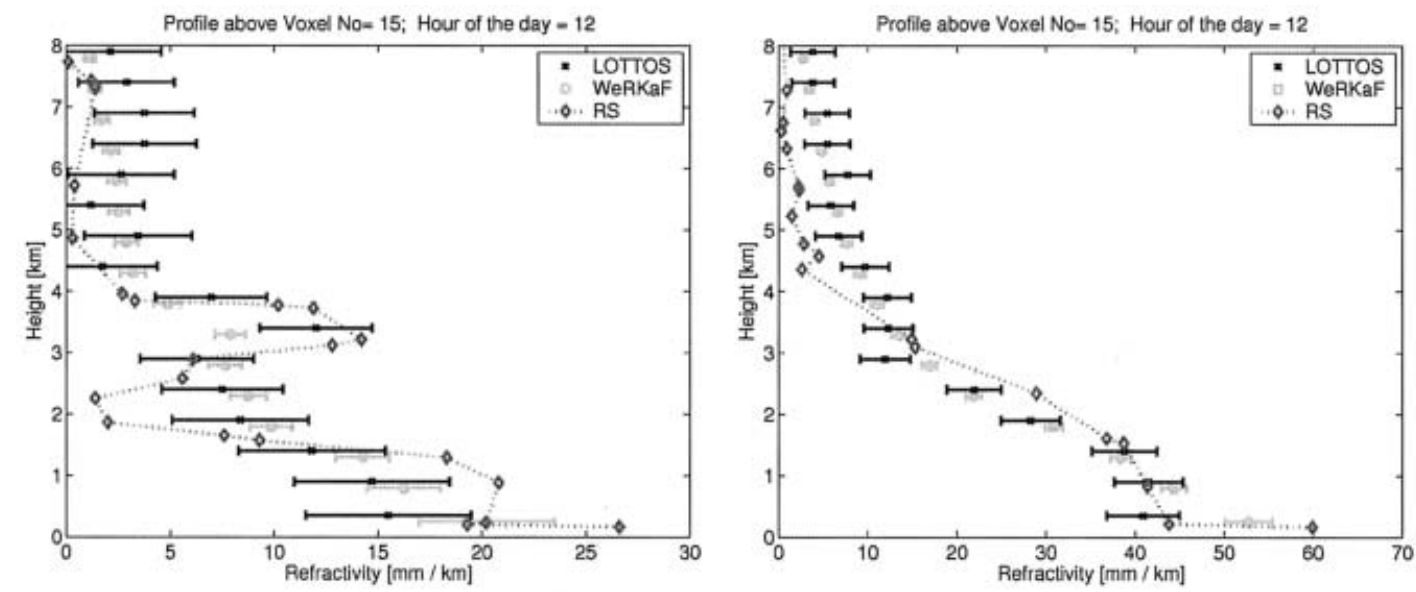

Fig. 6. Estimated refractivity from LOTTOS and WeRKaF for the $7^{\text {th }}$ (left), and the $9^{\text {th }}$ (right) of May 2002. Displayed are the solutions above voxel 15 at $12 \mathrm{UT}$, the time when the radiosondes (RS) are launched. The location of the RS launches is within voxel 15. The RMS errors by day are 7 , and $4.5 \mathrm{~mm} / \mathrm{km}$ for both methods.

In Fig. 6 we display results using real GPS data for $7^{\text {th }}$ and $9^{\text {th }}$ of May 2002. The voxel space used was $5 \times 5 \times 16$, producing a height resolution of $500 \mathrm{~m}$. One of the days had an inversion event (left) while the other (right) had a more regular feature. We present the 12 UT solution where the corresponding radiosonde profiles from Landvetter are also displayed. All real data results are based on the non-fiducial PPP solutions. The results again show the dif- ficulty of the solutions to capture the profiles irregularity (left) although able to more accurately follow the structure in the smoother profile case (right). Here we should point out that in the analyses of the real data the value of the height scaling constant was set to $C_{0} \ll 100$ approaching $\approx 1$. This had an effect of reduced allowed refractivity variability with height. Using the calculated $C_{0}=100$ will produce a very noisy solution due to the noise presence in the 
SD estimates. A need to constrain the allowed variability arose, which resulted in a reduced sensitivity to variations in height.

The ambition of this work was to assess the capabilities of one existing and one recently developed tomographic method. We mainly focused on the ability to resolve vertical structure and therefore all of the results addressed this issue. Since the tropospheric water vapor also has a horizontal structure and the tomographic methods do estimate a full 3-D state of the troposphere, the horizontal information should also be present in the results. Previous studies has already suggested how the information from a single GPS site, or from a small GPS network can be used for retrieval of linear horizontal gradients and gradient parameters (Elòsegui et al. 1999). Therefore we have not address the issue here. A difficulty with the studies of the horizontal refractivity structure is the lack of suitable data for verifications purposes.

\section{Conclusions}

Based on simulations, the suggested Kalman filter methods have good tomographic retrieval capabilities when a good geometry and accurate slant delay data are available. In the case of real data, the tomographic solutions are able to capture the general profile trend but fail to disclose the details of the profile. This is mainly due to the limits in the network geometry and the noise in the slant delays, which forces the use of tighter constraints in the Kalman filter setup. Using alternative methods for slant delay retrieval and adding observations from additional navigation systems will in the future offer better alternatives for 3-dimensional retrieval of water vapor refractivity.

\section{References}

Alber, C., R. Ware, C. Rocken, and J. Braun, 2000: Obtaining single path phase delays from GPS double difference. Geophys. Res. Lett., 27, 2661-2664.

Braun, J., C. Rocken, and R. Ware, 2001: Validation of line-of-sight water vapor measurements with GPS. Radio Sci., 36, 459-472.

Brown, R.G., and P.Y.C. Hwang, 1997: Introduction to Random Signals and Applied Kalman Filtering, John Wiley and Sons, 484pp.
Böhm, G., and A.L. Vesnaver, 1999: In quest of the grid, Geophysics, 64, 1116-1125.

Davis J.L., G. Elgered, A.E. Niell, and C.E. Kuehn, 1993: Ground-based measurements of gradients in the "wet" radio refractivity of air. $R a$ dio Sci., 28, 1003-1018.

Elòsegui P., J.L. Davis, L.P. Gradinarsky, G. Elgered, 1999: J.M. Johansson, D.A. Tahmoush, and A. Rius, Sensing atmospheric structure using small-scale space geodetic networks. Geophys. Res. Lett., 26, 2445-2448.

- and Davis, 2003: Accuracy assessment of slant path GPS signal delays, Proceedings of the International Workshop on GPS Meteorology —GPS Meteorology: Grand-Based and Space-Borne Applications-. (http://cfa-www. harvard.edu/space_geodesy/ATMSD/Publications/ atmsd_gpsmet03.pdf).

Emardson, T.R., Y. Bar-Sever, C. Naudet, J. Lijegren, 2000a: Measuring line-of-sight precipitable water vapor using GPS, Proceedings of the COST 716 Workshop: Towards Operational GPS Meteorology.

, J.M. Johansson, and G. Elgered, 2000b: The systematic behavior of water vapor estimates using four years of GPS observations. IEEE Trans. Geosci. Remote Sensing, 3, 324-329.

Flores, A., J.V.-G. de Arellano, L.P. Gradinarsky, and A. Rius, 2001: Tomography of the lower troposphere using a small dense network of GPS receivers. IEEE Trans. Geosci. Remote Sensing, 39, 439-447.

Gradinarsky, L.P., 2002: Sensing Atmospheric Water Vapor Using Radio Waves, Chalmers University of Technology, Technical Report No. 436, Ph-D thesis. (http://www.oso.chalmers.se/dwl/ lubo/Thesis.pdf).

and P.O. Jarlemark, 2002: GPS tomography using the permanent network in Göteborg: Simulations, Proceedings of the IEEE Positioning and Navigation Symposium, 128-133, (http:// www.oso.chalmers.se/dwl/lubo/AppendixC2.pdf). , J.M. Johansson, H.R. Bouma, H.-G. Scherneck, and G. Elgered, 2002: Climate monitoring using GPS. Phys. Chem. of the Earth, 27, $335-340$.

Jain, A.K., 1989: Fundamentals of Digital Image Processing, Prentice Hall, 529pp.

Niell, A., 1996: Global mapping functions for the atmosphere delay at radio wavelength. J. Geophys. Res., 101, 3227-3246.

Nolet, G., 1987: Seismic tomography with applications in global seismology and exploration geophysics, Dordrecht: Reidel.

Ruffini, G., A. Flores, A. Rius, 1998: GPS tomography of the ionospheric electron content with a cor- 
relation functional. IEEE Trans. Geosci. Remote Sensing, 36, 143-153.

Treuhaft, R.N., and G.E. Lanyi, 1987: The effect the dynamic wet troposphere on radio interferometric measurements. Radio Sci., 22, 251-265.
Zumberge, J.F., M.B. Heflin, D.C. Jefferson, M.M. Watkins, and F.H. Webb, 1997: Precise Point Positioning for the Efficient and Robust Analysis of GPS Data from Large Networks. J. Geophys. Res., 102, 5005-5017. 